\title{
Efectividad de las políticas públicas y actitudes. Análisis a través de la predisposición a participar en programas inclusivos en el entorno laboral
}

\section{Effectiveness of public policies and attitudes. Analysis through the willingness to participate in inclusive programs in the workplace}

\section{Resumen}

Para lograr una plena inclusión y desarrollo de las personas con discapacidad es imprescindible que participen en un entorno laboral normalizado, lo que, además de contribuir a mejorar su calidad de vida, produce un incremento de la cohesión social y del bienestar colectivo. A pesar de que el marco legislativo es cada vez más favorable a la discriminación positiva, existen múltiples factores que tienen lugar en el ámbito laboral y que condicionan el alcance y la efectividad de las políticas públicas a través de las medidas adoptadas. Un condicionante que se ha mostrado como elemento clave son las actitudes del entorno inmediato, en concreto de los compañeros y compañeras de trabajo, que son un aspecto fundamental para conseguir los objetivos propuestos. En esta investigación se analiza la intención que muestra el personal administrativo de participar en programas de apoyo a personas con discapacidad. Los datos analizados se obtienen a partir de una encuesta al personal de administración y servicios (PAS) de la Universidad de A Coruña. Los resultados demuestran que los valores sociales, las actitudes y la autopercepción de la propia capacidad para apoyar a las personas con discapacidad explican en gran medida dicha intención, lo que condiciona la efectividad de las políticas aplicadas.

\section{Palabras clave}

Políticas inclusivas, empleo, discapacidad, actitudes, inclusión.

\begin{abstract}
To achieve full inclusion and personal development of people with disabilities it is essential that they participate in a normalized working environment. This participation not only helps to improve their quality of life, but also produces an increase in social cohesion and collective welfare. Although the legislative framework is increasingly favorable to affirmative action, there are multiple factors at the workplace that determine the scope and effectiveness of the public policies. The attitudes of the coworkers has proven to be a key condition for achieving the goal of inclusion. The intention of the administrative staff to participate in programs that support people with a disability is discussed in this research. The data analyzed were obtained from a survey made among workers in administration and services (ASW) at the University of A Coruña. The results of the survey demonstrate that social values, attitudes and selfperception of one's ability to support people with a disability largely explain this intention. Thus, the intention determines the effectiveness of the policies.
\end{abstract}

\section{Keywords}

Inclusive policies, employment, disability, attitudes, inclusion.

\author{
Isabel Novo Corti \\ <isabel.novo.corti@udc.es> \\ Catedrática EU. Departamento de \\ Análisis Económico y Administración \\ de Empresas. Universidad de A Coruña
}

\section{Patricia Becerra Amado} <ecopbao2@udc.es>

Estudiante de doctorado. Universidad de A Coruña

\section{María Dolores López \\ <m.lopez@udc.es>}

Técnico de gestión del Servicio de Personal. Universidad de A Coruña

Para citar:

Novo Corti, I.; Becerra Amado, P. y López, M.D. (20I4): "Efectividad de las políticas públicas y actitudes. Análisis a través de la predisposición a participar en programas inclusivos en el entorno laboral", Revista Española de Discapacidad, 2 (2): 207-2 I 8.

$<$ http://dx.doi.org/IO.5569/23405104.02.02.II>

Fecha de recepción: I4-09-20I3 Fecha de aceptación: 29-09-20I4 


\section{Introducción}

La exclusión social es el proceso mediante el cual los individuos son total o parcialmente privados de una participación plena en la sociedad en la que viven (European Foundation, I995). Las necesidades personales, sociales, económicas, culturales y políticas se definen como derechos, esto significa que la exclusión debe definirse como la imposibilidad o incapacidad de ciertas personas de acceder a estos derechos a no ser que dispongan de algún tipo de ayuda externa.

El acceso a un puesto de trabajo es un factor clave para la inclusión laboral de las personas y en consecuencia de su plena realización en la sociedad, ya que no sólo supone el acceso a los recursos económicos, sino también al establecimiento de redes sociales, de ahí que la carencia de empleo pueda llevar a la exclusión social. El desempleo es un componente clave que incrementa en gran medida las posibilidades de sufrir exclusión (Escarbajal e Izquierdo, 20I3). Este riesgo no es el mismo para toda la sociedad, pues hay ciertos colectivos que pueden caer en él con mayor facilidad, precisamente porque en ellos concurren varias posibles causas de exclusión, lo que comúnmente se denomina exclusión múltiple. Tal es el caso de las personas con discapacidad, ya que por el mero hecho de tenerla carecen de las mismas facilidades de socialización que quienes no la tienen, aunque, naturalmente, los grados varían en función de la naturaleza e intensidad de la misma. El concepto de discapacidad va evolucionando como resultado de la interacción entre las personas con deficiencias y las barreras debidas a la actitud y al entorno, que evitan su participación plena y efectiva en la sociedad, en igualdad de condiciones con las demás (ONU, 2006).

La discapacidad también resulta un condicionante negativo en el acceso al empleo, especialmente al normalizado, entendiendo como tal el que se desempeña en centros de trabajo no específicos para personas con discapacidad, aunque para el desarrollo del trabajo sea necesario realizar algunas adaptaciones en ciertos casos. En general, se puede afirmar que la participación en el mercado de trabajo tiene importantes efectos en el nivel y la calidad de vida de la población en general $\mathrm{y}$, en particular, del colectivo de personas con discapacidad (De Lorenzo, 2004).

La capacidad para integrar a personas con condicionantes distintos, ofreciéndoles oportunidades para realizar con éxito y en igualdad un proyecto de vida digno y justo es uno de los mejores indicadores del grado de desarrollo alcanzado por una sociedad (Pereda, De Prada y Actis, 20 I 2). El colectivo de personas con discapacidad constituye un eje de actuación prioritario en las políticas sociales y uno de sus fines esenciales es la integración laboral de dichas personas. Actuaciones como los Objetivos del Milenio o la Estrategia 2020 son llevadas a cabo para tratar de reducir las desigualdades y aumentar el bienestar social. La mayor parte de los países desarrollados son sensibles a esta cuestión y por tal motivo han elaborado medidas para promover la inclusión sociolaboral de las personas con discapacidad. Como ejemplos de estas medidas cabe mencionar la Convención Internacional sobre los Derechos de las Personas con Discapacidad y su Protocolo Facultativo aprobados el $\mathrm{I} 3$ de diciembre de 2006 por la Asamblea General de las Naciones Unidas (ONU, 2006), en la que se recogen los derechos de las personas con discapacidad así como las obligaciones de los Estados Parte de promover, proteger y asegurar esos derechos. España la adaptó y se publicó en el Boletín Oficial del Estado de 2 I de abril de 2008. La adaptación y modificación de diversas normas para hacer efectivos los derechos que la Convención proclama y garantiza se hace mediante el Real Decreto I276/20 I I, de I6 de septiembre, de adaptación normativa a la Convención Internacional sobre los derechos de las personas con discapacidad.

Teniendo en cuenta la importancia del entorno laboral para una exitosa inclusión de las personas con discapacidad, nos hemos centrado en analizar la intención que tienen los compañeros de trabajadores con discapacidad 
en participar en programas de apoyo a la inclusión; esta variable nos permite conocer la predisposición de la persona a trabajar con compañeros con discapacidad y su estudio en profundidad nos muestra qué variables pueden afectar de forma más específica en ella; esto facilita el planteamiento de políticas sociales y laborales concretas encaminadas a la modificación del comportamiento de esas variables y en consecuencia que la predisposición del individuo a aceptar a ese colectivo en su entorno laboral varíe de forma positiva. Se trata de una primera aproximación a través de la realización de una encuesta entre I Io trabajadoras y trabajadores administrativos en la Universidad pública de A Coruña.

Inicialmente se realiza una breve revisión de la literatura académica relacionada con el tema que nos ocupa, a continuación se explica la metodología utilizada. En la sección 5 se presentan los resultados obtenidos, en la sección 6 la discusión de los mismos y se finaliza con unas breves conclusiones y recomendaciones de posibles políticas de actuación.

\section{La discapacidad y su relación con el} riesgo de exclusión

Sabemos que la inclusión social está estrechamente relacionada con la capacidad de obtener recursos materiales (trabajo=dinero) que evitan caer en la pobreza, además de que con ella se establecen lazos sociales de relación, que van desde los más cercanos con la familia hasta las comunidades de vecinos, municipios, y abarcan la intervención en los mecanismos de participación individual en la toma de decisiones colectivas, como puede ser a través de la elección de representantes en distintos foros (votaciones). Las personas con discapacidad tienen dificultades especiales relacionadas con su condición que afectan a todas o alguna de las esferas de su vida y por tanto corren el peligro de un mayor riesgo de exclusión que quienes no la tienen. Es frecuente que las personas con discapacidad accedan al mercado laboral mediante centros especiales de empleo y no a través del empleo ordinario ${ }^{\mathrm{I}}$, que genera un grado de inclusión social mucho mayor, precisamente, porque en ese entorno laboral participan gran diversidad de personas, que pueden tener o no una discapacidad.

Pero existen dificultades añadidas de acceso al mercado de trabajo; es habitual que la discapacidad vaya ligada con la pertenencia a grupos de mayor edad, lo que incrementa el riesgo de exclusión laboral de ese colectivo. Tal como señala el Instituto Nacional de Estadística de España (INE), se ha comprobado la existencia de una estrecha relación entre edad y discapacidad, de modo que a mayor edad corresponde mayor porcentaje de personas con discapacidad. Esto afecta a la estructura de la población con discapacidad en el mercado de trabajo, que es de mayor edad que para las personas que no tienen discapacidad (Tabla I), y nos sitúa en un marco de acción en el que confluyen al menos dos factores que podrían ser considerados como de riesgo de exclusión: la discapacidad y la edad.

Tabla 1. Población ocupada según discapacidad por grupo de edad (2012)

\begin{tabular}{|l|c|c|c|c|}
\hline \multirow{2}{*}{ Edad } & \multicolumn{2}{|c|}{$\begin{array}{c}\text { Personas sin } \\
\text { discapacidad }\end{array}$} & \multicolumn{2}{c|}{$\begin{array}{c}\text { Personas con } \\
\text { discapacidad }\end{array}$} \\
\cline { 2 - 5 } & $\begin{array}{c}\text { Personas } \\
\text { (Miles) }\end{array}$ & $\%$ & $\begin{array}{c}\text { Personas } \\
\text { (Miles) }\end{array}$ & $\%$ \\
\hline $16-24$ & 826,6 & 4,9 & 7,9 & 2,2 \\
\hline $25-34$ & $4.188,6$ & 25,0 & 46,0 & 12,9 \\
\hline $35-44$ & $5.263,1$ & 31,4 & 96,0 & 27,0 \\
\hline $45-54$ & $4.297,1$ & 25,6 & 118,1 & 33,2 \\
\hline $55-64$ & $2.192,7$ & 13,1 & 87,5 & 24,6 \\
\hline
\end{tabular}

Fuente: Elaboración propia a partir de datos INE.

I. Cuando se habla de empleo ordinario se hace referencia al empleo que tiene lugar en empresas que no son Centros Especiales de Empleo, aquellos en los que el porcentaje mayoritario de trabajadores lo conforman personas con discapacidad (INE, 20I3). 
La inclusión social como meta importante para la sociedad española está recogida en la Carta Magna de I978, que en su artículo I.I establece la igualdad como uno de los valores superiores del ordenamiento jurídico del Estado social y democrático de Derecho español y que todos los españoles son iguales ante la ley, sin que pueda prevalecer discriminación alguna por razón de nacimiento, raza, sexo, religión, opinión o cualquier otra condición o circunstancia personal o social (artículo I4). Por otra parte, la Constitución Española hace patente la necesidad de promover las condiciones para garantizar la libertad y la igualdad real y efectiva del individuo y de los grupos en que se integra, a través de la eliminación de los obstáculos que la impidan (artículo 9.2), indicando que los poderes públicos deberán realizar una política de integración social para las personas con discapacidad que permita "el disfrute de los derechos que este Título otorga a todos los ciudadanos" (artículo 49), como el derecho al trabajo, a la libre elección de profesión u oficio, a la promoción a través del trabajo y a una remuneración suficiente para satisfacer sus necesidades y las de su familia, sin que en ningún caso pueda hacerse discriminación por razón de sexo (artículo 35.I), complementados en el artículo 40, con la recomendación de realizar políticas orientadas al pleno empleo y de fomento de la formación profesionales.

Este tema resultó ya muy preocupante en el pasado, tanto es así que la Organización Mundial de la Salud y el Grupo del Banco Mundial han realizado conjuntamente un Informe mundial sobre la discapacidad, para proporcionar datos destinados a la formulación de políticas y programas innovadores que mejoraran las vidas de las personas con discapacidades y facilitaran la aplicación de la Convención de Naciones Unidas sobre los Derechos de las Personas con Discapacidad, que entró en vigor en mayo de 2008.

\subsection{El nivel educativo como facilitador de acceso al mercado laboral}

El hecho de que las tasas de actividad y empleo aumenten a medida que se incrementa el nivel de formación, pone de manifiesto que se trata de una variable integradora de primera magnitud y de ahí que según el nivel de estudios se encuentren notables diferencias respecto a la población sin discapacidad (para los datos mostrados por el INE en 20I2). En la comparativa entre personas con discapacidad y personas sin discapacidad, encontramos que son los primeros los que acceden en mucha menor medida a los estudios superiores: sólo el $27,4 \%$ de las personas con discapacidad tiene estudios superiores frente al 39,7\% del resto de la población, pero, además, en los niveles más bajos también se detecta que el porcentaje de personas con estudios primarios ( ( $8,8 \%$ ) casi duplica al de personas sin discapacidad $(9,5 \%)$, lo que pone también de manifiesto el menor grado de formación de las personas con discapacidad, configurando una estructura del mercado de trabajo diferente para ambos colectivos. Ello nos hace recalcar nuevamente el otro posible aspecto generador de riesgo de exclusión señalado: educación (Tabla 2).

Se ha comprobado que la tasa de actividad de la población de personas con discapacidad aumenta con el nivel de formación, lo que se convierte en una herramienta imprescindible para recortar diferencias respecto a las personas sin discapacidad. Para datos de $20 \mathrm{I} 2$ la tasa de actividad de las personas con discapacidad con estudios secundarios es del $56,2 \%$, que es 36,2 puntos superior a la tasa de actividad de quienes tienen estudios primarios. El nivel de estudios influye, por tanto, de forma importante en la empleabilidad de las personas con discapacidad.

\subsection{Medidas para la promoción del empleo específicas para personas con discapacidad}

Un importante paso en cuanto a medidas promotoras de empleo para las personas con discapacidad fue la $\mathrm{LISMI}^{2}$ en la que se puso de manifiesto que la inclusión social de las personas con discapacidad debe pasar necesariamente

2. Ley I3/1982, de integración social de los minusválidos. Actualmente derogada por el Real Decreto Legislativo I/20I3, por el que se aprueba el Texto Refundido de la Ley General de derechos de las personas con discapacidad y de su inclusión social. 
Tabla 2. Población según discapacidad y nivel estudios (2012)

\begin{tabular}{|l|c|c|c|c|}
\hline \multirow{2}{*}{ Nivel de Estudios } & \multicolumn{2}{|c|}{$\begin{array}{c}\text { Personas sin } \\
\text { discapacidad }\end{array}$} & \multicolumn{2}{c|}{$\begin{array}{c}\text { Personas con } \\
\text { discapacidad }\end{array}$} \\
\cline { 2 - 5 } & $\begin{array}{c}\text { Personas } \\
\text { (Miles) }\end{array}$ & $\%$ & $\begin{array}{c}\text { Personas } \\
\text { (Miles) }\end{array}$ & $\%$ \\
\hline Analfabetos & 36,8 & 0,2 & 3,3 & 0,9 \\
\hline Primaria & $1.597,7$ & 9,5 & 66,8 & 18,8 \\
\hline Secundaria y programas de formación e inserción laboral & $8.472,2$ & 50,5 & 187,8 & 52,8 \\
\hline Superiores, incluyendo doctorado & $6.661,5$ & 39,7 & 97,5 & 27,4 \\
\hline
\end{tabular}

Fuente: Elaboración propia a partir de datos INE.

por la integración en el mercado laboral; pero en la actualidad se ha intentado aportar un marco normativo más dinámico que aporte nuevas soluciones: en este sentido se redactó la Estrategia global de acción para el empleo de personas con discapacidad 2008-20I2, elaborado con la participación de organizaciones sindicales y empresariales y con las asociaciones representativas del sector de la discapacidad y sus familias, así como con centros directivos del antes denominado Ministerio de Trabajo y Asuntos Sociales y ahora integrados en el Ministerio de Empleo y Seguridad Social, el Ministerio de Educación, Cultura y Deporte y el Ministerio de Sanidad, Servicios Sociales e Igualdad, diversos departamentos ministeriales y las Comunidades Autónomas. Esta actuación nació con vocación de acción continua y diálogo permanente y tuvo como objetivo abordar la política de empleo y la legislación laboral en un contexto amplio, en el que se contemplan otros ámbitos relacionados con ellas, como la educación, la seguridad social, la política fiscal, etc.

Los resultados de las medidas de apoyo al empleo de las personas con discapacidad se aprecian en los datos de 20I2, ya que los trabajadores con discapacidad del sector privado pertenecientes a empresas con más de 50 trabajadores (afectadas por la LISMI) suponen un $2 \%$ del total de asalariados de dichas empresas, mientras que en las de menos de 50 trabajadores sólo suponen el I, $5 \%$. Pero estas medidas no sólo han de favorecer el empleo ordinario de este colectivo, sino que deben tratar de favorecer la contratación indefinida. Una de las medidas más extendida es la de reducciones y bonificaciones en las cuotas de cotización, tanto para trabajadores asalariados como por cuenta propia o las modalidades de contrato específico o a través de las cuotas de reserva. Otra medida de fomento para el empleo ordinario en el caso de personas asalariadas, es la modalidad de contrato específico para trabajadores con discapacidad en el que se combina bonificaciones en las cuotas de cotización a la Seguridad Social con ciertas subvenciones (según el INE, en el año 2012 el $24,9 \%$ de los asalariados con discapacidad tienen la modalidad de contrato específico de discapacidad).

Por lo que se refiere a la cuota de reserva como medida de promoción del empleo ordinario, las empresas públicas y privadas de más de 50 trabajadores deben dar empleo al menos a un 2,0\% de trabajadores con discapacidad, aunque se contempla la posibilidad de realización de algún tipo de media alternativa al cumplimiento de dicha cuota de reserva. En el caso de la Administración Pública, este porcentaje de cuota de reserva es del 7,0\%. El sector público atrae más empleo de personas con discapacidad que el privado, lo que apunta hacia posibles patrones de comportamiento diferentes.

En este sentido, conviene reflexionar sobre las actitudes y la influencia sobre esta medida de promoción de empleo. Si bien en el sector público, se procede al establecimiento de la cuota, en el sector privado no es infrecuente que 
las empresas opten por medidas alternativas, que, si bien apoyan al empleo de las personas con discapacidad, no generan efectos tan inmediatos como la aplicación de la cuota. La pregunta pertinente en este momento es ¿Qué lleva a los empresarios a optar por medidas alternativas en lugar de cumplir la cuota de reserva? En nuestra opinión hay dos factores principales. El primero es relativamente fácil de detectar e incluso de cuantificar: los costes de adecuación del puesto de trabajo, que en muchas ocasiones es necesaria para poder adaptarse a las necesidades específicas que presente el trabajador. El otro factor permanece oculto y radica en los temores de las posibles distorsiones que una persona con discapacidad puede introducir en el ambiente laboral, ya que, como persona singular y diferente, podría alterar los comportamientos o relaciones habituales entre compañeros: estamos en el terreno de los valores, las normas sociales y las actitudes individuales, junto con la autopercepción del control de la situación, que consideramos claves para comprender la intención de apoyo (o no) a los compañeros con discapacidad, por parte de sus colegas; estos elementos están recogidos por la "Teoría del Comportamiento Planeado" (Ajzen, I99I). En este sentido, existe evidencia empírica contrastada en trabajos como MuñozCantero, Novo-Corti y Rebollo Quintela (20I3) que han aplicado esta teoría al entorno laboral privado y han comprobado que las actitudes son un punto débil que deberá ser reforzado para conseguir entornos laborables favorables y, consecuentemente, evitar los temores a la presencia de personas con discapacidad en los entornos laborales ordinarios.

Son numerosas las diferencias existentes entre el sector público y el privado, por lo que consideramos que los entornos laborales de los mismos son difícilmente equiparables, y, por ello, nos hemos preguntado si en el sector público ocurriría lo mismo que lo que ocurría en el sector privado en la investigación que acabamos de mencionar. Para responder a esta pregunta, hemos replicado el cuestionario de Muñoz-Cantero et al. (2OI3) y lo hemos aplicado en el entorno del personal funcionario y laboral de la Universidad de A Coruña.

\section{El entorno laboral como factor clave para} la inclusión efectiva

La revisión de la literatura científica, realizada en la sección 2, apunta hacia la importancia del entorno laboral como factor clave para una inclusión laboral normalizada exitosa. De ahí el interés en evaluar los factores de que dependen las actitudes y la intención de apoyo a las personas con discapacidad, empleadas en el mismo centro de trabajo. Para ello se ha tomado como variable a explicar la intención de participar en programas de apoyo a las personas con discapacidad, formulada a través de la afirmación "Si en mi centro de trabajo se propusiese programas de apoyo a las personas con discapacidad, yo me apuntaría”. Esta ha sido, por tanto, la variable dependiente en este trabajo. Las hipótesis a contrastar han sido las siguientes:

- Hor: La intención positiva de apoyo a las personas con discapacidad por parte de sus compañeros en el centro de trabajo no influye en la intención de participar en programas de apoyo propuestos por la Administración Pública.

- Ho2: El sentimiento de obligación de ayudar a otras que las necesitan no influye en la intención de participar en programas de apoyo propuestos por la Administración Pública.

- Ho3: La percepción de estar preparado para ayudar o apoyar a una persona con discapacidad no influye en la intención de participar en programas de apoyo propuestos por la Administración Pública.

- Ho4: Sentirse gratificado al dedicar algo de tiempo propio en ayudar a los demás no influye en la intención de participar en programas de apoyo propuestos por la Administración Pública. 


\section{Metodología}

Se ha realizado un análisis de regresión por mínimos cuadrados ordinarios (MCO), si bien conviene aclarar que aunque la variable dependiente es ordinal, tipo Likert, se le ha dado un tratamiento como variable cuantitativa; de este modo se justifica la estimación MCO frente a otros modelos de análisis de regresión como los logit o probit, especialmente indicados para el tratamiento de las variables discretas, que hacen posible la modelización de variables cualitativas.

Los datos utilizados en la regresión provienen de las respuestas del personal de administración y servicios (PAS), funcionario y contratado de la Universidad de A Coruña (España) a una encuesta realizada en 20 I I. La recogida de datos ha sido presencial, en papel, cumpliendo los requisitos de confidencialidad y anonimato, aunque se realizasen en presencia del responsable de la recogida de la información. Se les explicó a todos los participantes el objetivo de la investigación y se pidieron respuestas de total sinceridad.

El cuestionario utilizado está basado en NovoCorti, Muñoz-Cantero y Calvo-Porral (20I I) y ha sido adaptado para esta investigación (se seleccionó un ítem como variable explicada y dependiente a su vez de otros seis). Se ha utilizado una escala tipo Likert en la que las respuestas se valoraban de I al 5 ; los ítems fueron valorados por los participantes que indicaron su grado de conformidad o disconformidad con lo que se exponía en cada afirmación (donde I representa total desacuerdo y 5 completo acuerdo con la afirmación enunciada). Las variables de la regresión se muestran en el siguiente esquema:

Variable dependiente: Si en mi centro de trabajo se propusiesen programas de apoyo a las personas con discapacidad, yo me apuntaría. 수

Variables independientes o explicativas:

I. Tengo intención de ayudar a la inclusión de las personas con discapacidad.
2. Creo que la obligación de cualquier persona es ayudar a otra que lo necesite.

3. Me siento capacitado/a para apoyar y ayudar a cualquier persona con discapacidad.

4. Creo que es muy gratificante dedicar algo de tiempo propio en ayudar a los demás.

La muestra se ha realizado a I Io trabajadores de la Universidad de A Coruña, de una media de edad de 43,45 años y de las que un elevado porcentaje $(45 \%)$, tenía alguna persona con discapacidad en la familia, un dato a tener en cuenta a la hora de analizar los resultados (Tabla 3).

Tabla 3. Estructura de la muestra

\begin{tabular}{|l|l|c|c|}
\hline \multicolumn{2}{|l|}{ Variable } & $\begin{array}{c}\mathbf{N}^{\circ} \\
\text { personas }\end{array}$ & $\%$ \\
\hline \multirow{2}{*}{ Sexo } & Hombres & 39 & 35,5 \\
\cline { 2 - 4 } & Mujeres & 71 & 64,5 \\
\hline \multirow{2}{*}{ Tiene discapacidad } & $\mathrm{Si}$ & 7 & 6,4 \\
\cline { 2 - 4 } & $\mathrm{No}$ & 103 & 93,6 \\
\hline \multirow{2}{*}{$\begin{array}{l}\text { Tiene algún familiar } \\
\text { con discapacidad }\end{array}$} & $\mathrm{Si}$ & 45 & 40,9 \\
\cline { 2 - 4 } & $\mathrm{No}$ & 65 & 59,1 \\
\hline
\end{tabular}

Fuente: Elaboración propia a partir de los resultados obtenidos de la muestra.

De las cuestiones planteadas se seleccionan los ítems objeto de estudio y se realiza un análisis de regresión cuyos resultados se recogen en la Tabla 4, donde recordemos que la variable dependiente (la que queremos explicar) es la intención de participar en los programas de apoyo a las personas con discapacidad, valorada a través de la pregunta "Si en mi centro de trabajo se propusiesen programas de apoyo a las personas con discapacidad, yo me apuntaría” y las variables independientes/explicativas (son las variables que explican la dependiente) recogen las intenciones de los trabajadores a apoyar la inclusión de las personas con discapacidad ("Tengo intención de ayudar a la inclusión de las personas con discapacidad"), las actitudes en general ("Creo que la obligación de cualquier 
persona es ayudar a otra que la necesite" y "Creo que es muy gratificante dedicar algo de tiempo propio a la ayuda a los demás”) y la autopercepción de la capacidad de ayudar a las personas con discapacidad ("Me siento capacitada/o para apoyar y ayudar a cualquier persona con discapacidad").

\section{Resultados}

Del análisis de regresión se comprueba que todas las variables son significativas $(\mathrm{p}<0,05$, véase Tabla 4) y se refuerzan los resultados obtenidos en el trabajo de Novo-Corti y López (presentado en el Congreso REPS, celebrado en la Universidad de Alcalá, Madrid, 20I3). Las autoras proponen la aplicación del modelo de TPB y comprueban que las variables latentes norma social, actitud y control percibido son claves para explicar la intención de apoyo a los compañeros con discapacidad en el entorno laboral de los funcionarios; del mismo modo, en nuestra investigación queremos exponer cuáles son los factores que condicionan el compromiso del agente a querer participar en programas que fomenten la inclusión y las variables independientes se muestran como elementos clave para ello, ya que las intenciones, los valores personales o actitudes y los valores sociales o normas dominantes, junto con la autopercepción de la capacidad para acometer la acción consiguen explicar el $50 \%$ de la variable dependiente; y actuando sobre ellas se pueden fomentar entornos laborales más inclusivos. La capacidad explicativa de este modelo indica que las variables señaladas pueden predecir el comportamiento de la variable "intención de participar en las actividades propuestas por la empresa para apoyar la inclusión laborar de personas con discapacidad" (medida a través de la pregunta "Si en mi centro de trabajo se propusiese programas de apoyo a las personas con discapacidad, yo me apuntaría") alcanza un valor muy próximo al 50\%, logrando que las variables independientes expliquen cerca de la mitad de la variable dependiente (valor de R cuadrado ajustado = 0,446). El valor del estadístico Durvin Watson es de I,882 y el R cuadrado de 0,466 .

La relación entre la variable dependiente y las independientes se puede ver de forma esquemática en los gráficos I a 4 , que recogen la relación entre la variable dependiente y cada una de las variables independientes.

\section{Tabla 4. Análisis de regresión lineal por MCO}

\begin{tabular}{|l|c|c|c|c|c|}
\hline \multirow{2}{*}{$\begin{array}{l}\text { Variable dependiente: } \\
\text { Si en mi centro de trabajo se propusiese } \\
\text { programas de apoyo a las personas con } \\
\text { discapacidad, yo me apuntaría }\end{array}$} & \multicolumn{2}{|c|}{$\begin{array}{l}\text { Coeficientes no } \\
\text { estandarizados }\end{array}$} & $\begin{array}{c}\text { Coeficientes } \\
\text { tipificados }\end{array}$ & & \multirow{2}{*}{ Sig. } \\
\cline { 2 - 5 } & B & Error típ. & Beta & t & \multirow{2}{*}{0} \\
\hline $\begin{array}{l}\text { Tengo intención de ayudar a la inclusión de las } \\
\text { personas con discapacidad }\end{array}$ & 0,290 & 0,083 & 0,297 & 3,510 & \multirow{2}{*}{001} \\
\hline $\begin{array}{l}\text { Creo que la obligación de cualquier persona es } \\
\text { ayudar a otra que la necesite }\end{array}$ & 0,302 & 0,095 & 0,270 & 3,171 & 0,002 \\
\hline $\begin{array}{l}\text { Me siento capacitada/o para apoyar y ayudar a a } \\
\text { cualquier persona con discapacidad }\end{array}$ & 0,249 & 0,077 & 0,256 & 3,245 & 0,002 \\
\hline $\begin{array}{l}\text { Creo que es muy gratificante dedicar algo de } \\
\text { tiempo propio en ayudar a los demás }\end{array}$ & 0,137 & 0,088 & 0,122 & 1,561 & 0,121 \\
\hline
\end{tabular}

Fuente: Elaboración propia a partir de los resultados obtenidos de la regresión con SPSS. 


\section{Discusión de resultados}

Los resultados indican que para las tres primeras variables explicativas se rechazan las hipótesis nulas de partida, por lo que el comportamiento de esas variables sí que influye en la dependiente de forma significativa. Sin embargo, para el caso de "Creo que es muy gratificante dedicar algo de tiempo propio en ayudar a los demás” se acepta la hipótesis nula (Ho4: Sentirse gratificado al dedicar algo de tiempo propio en ayudar a los demás no influye en la intención de participar en programas de apoyo propuestos por la Administración Pública) provocando que la satisfacción a la hora de ayudar a los demás se muestre irrelevante para la variable dependiente. Las personas ayudan a los demás movidos por valores, creencias, etc., no por el mero hecho de sentirse mejor al hacerlo.

Además, la variable que muestra un coeficiente más elevado es "Creo que la obligación de cualquier persona es ayudar a otra que la necesite", lo que pone de manifiesto la importancia de los valores transmitidos por la sociedad, que

Gráfico 1. Variable dependiente: Si en mi centro de trabajo se propusiese programas de apoyo a las personas con discapacidad, yo me apuntaría
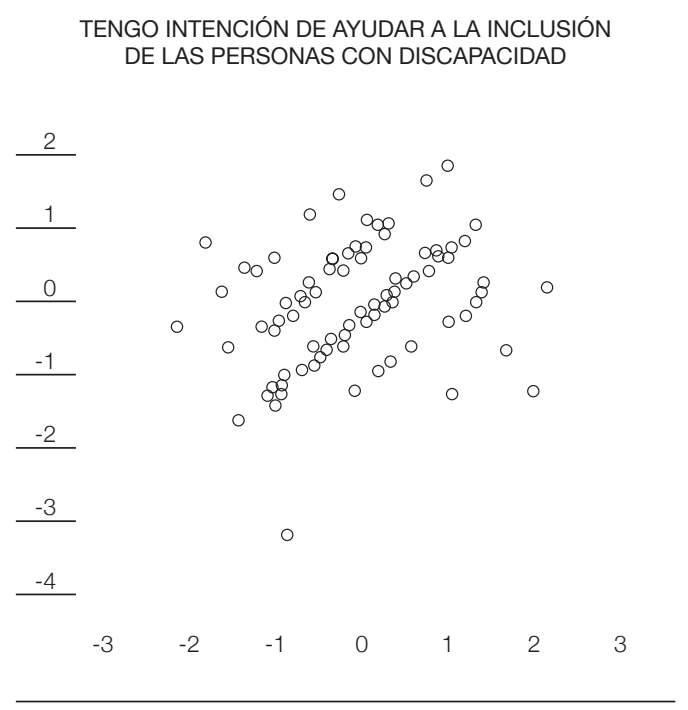

hace internalizar a las personas la obligación de ayudarse entre sí. En el Gráfico 2 se representa la relación de esta variable con la intención de participar en los programas de apoyo a la discapacidad propuestos en el centro de trabajo y se aprecia una relación claramente positiva.

La autopercepción de la capacidad para ayudar (o control percibido) se muestra como una variable importante para el compromiso en los programas de apoyo promovidos en la empresa (en nuestra investigación la medimos a través de la variable "Me siento capacitado/a para apoyar y ayudar a cualquier persona con discapacidad"), tal como se refleja en el Gráfico 3, indicando con claridad que cuanto mayor es la capacidad percibida, mayor es la predisposición a comprometerse en dichos programas.

Por último, el comportamiento de la variable "Creo que es muy gratificante dedicar algo de tiempo propio a la ayuda a los demás” es importante para explicar la participación en las acciones inclusivas promovidas por la empresa, con correlaciones positivas, tal como se muestra en el Gráfico 4.
Gráfico 2. Variable dependiente: Si en mi centro
de trabajo se propusiese programas de apoyo a
las personas con discapacidad, yo me apuntaría

CREO QUE LA OBLIGACIÓN DE CUALQUIER PERSONA ES AYUDAR A OTRA QUE LA NECESITE

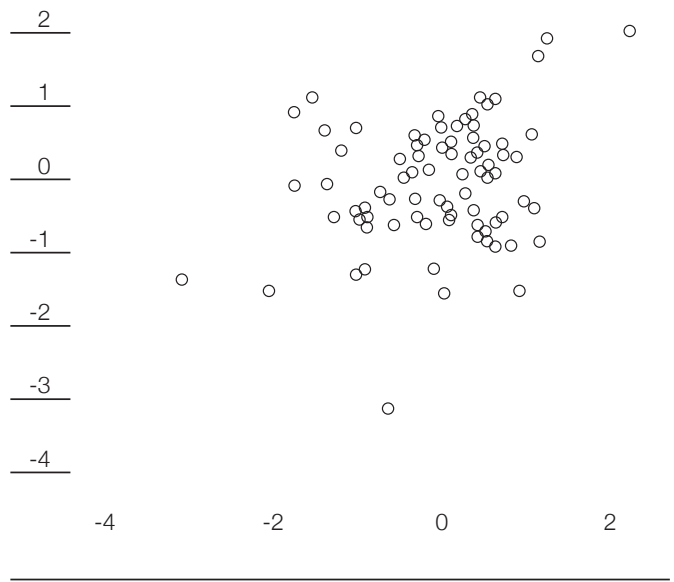


Gráfico 3. Variable dependiente: Si en mi centro
de trabajo se propusiese programas de apoyo a
las personas con discapacidad, yo me apuntaría

ME SIENTO CAPACITADA/O PARA APOYAR Y AYUDAR A CUALQUIER PERSONA CON DISCAPACIDAD

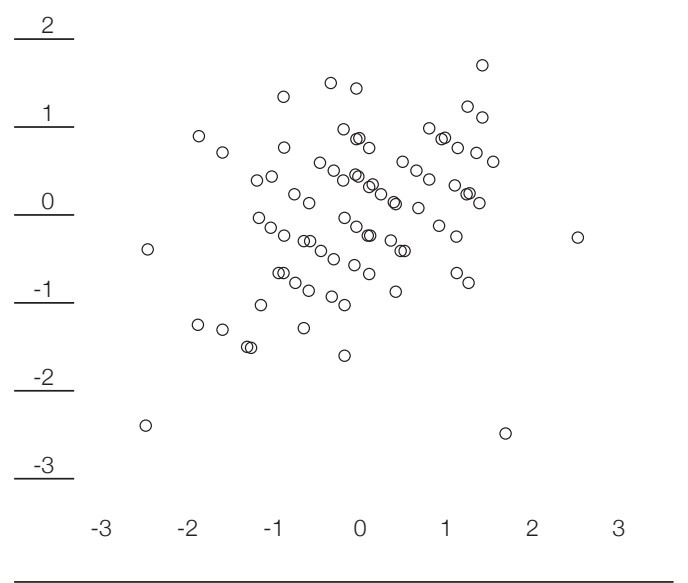

Finalmente, la intención que tiene cada persona de ayudar a personas con discapacidad es una de las variables con coeficientes positivos más elevados (Gráfico I), como corresponde con los propios intereses y deseos. En este punto conviene recordar los resultados de los trabajos citados anteriormente, de dónde se comprueba, mediante la "Teoría del Comportamiento Planeado" (Ajzen, I99I), que las intenciones (variable independiente en nuestro caso) están explicadas por las actitudes, control percibido y normas sociales, que en esta investigación se expresan a través de las variables explicativas.

\section{Conclusiones y recomendaciones}

Las principales conclusiones, en concordancia con la teoría, indican que la autopercepción de la capacidad es una variable clave para desear participar en programas inclusivos en el entorno laboral, por tanto se recomienda que se realicen acciones formativas en dicho sentido, como paso previo a programas concretos. Consideramos
Gráfico 4. Variable dependiente: Si en mi centro de trabajo se propusiese programas de apoyo a las personas con discapacidad, yo me apuntaría

CREO QUE ES MUY GRATIFICANTE DEDICAR ALGO DE TIEMPO PROPIO EN AYUDAR A LOS DEMÁS

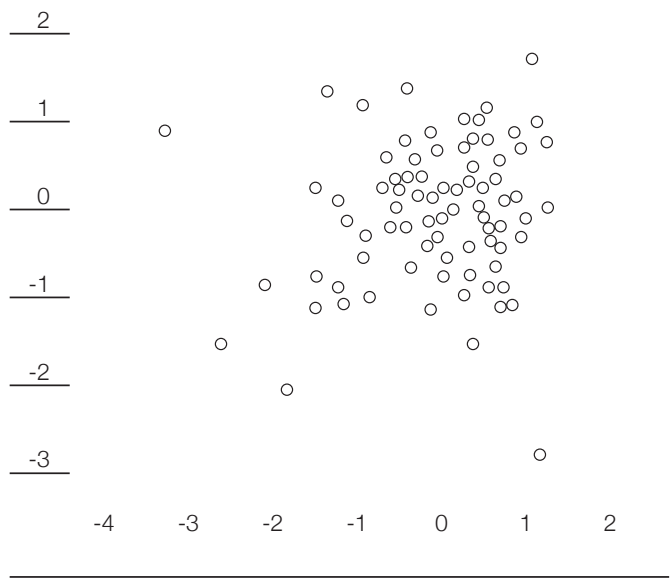

que si se incrementa la percepción de control de la situación por parte del agente, esto repercutirá en su posible miedo a ayudar a una persona con discapacidad y ese dominio le permitirá aceptar a este colectivo sin ningún tipo de dificultad y de modo totalmente normalizado.

Por otra parte, dado que las normas sociales, las actitudes y las intenciones formadas a partir de las mismas son, asimismo, variables determinantes, sugerimos que se incorporen de forma transversal en todas las acciones que se realicen en la empresa, para generar, impulsar o reforzar actitudes individuales y colectivas favorables hacia las personas con discapacidad. Así, si se consigue generar una conciencia positiva global en la empresa hacia la discapacidad, el trabajador que presente algún tipo de dificultad será ayudado por sus compañeros sin que lo puedan considerar una carga o pérdida de tiempo. Se refuerza la sensibilización y el trabajo en equipo.

Se sugiere acometer acciones tendentes a promover actitudes más positivas, aumentar el control percibido, en cuanto a capacidades de apoyo a las personas con discapacidad por parte 
de quienes no la tienen y seguir reforzando las normas sociales dominantes, concienciando a la población en general y a quienes están más cercanos a las personas con discapacidad, en particular, de la importancia de mantener una sociedad cohesionada que ayude a aquellos que necesitan apoyos. Sobre todo a los que se encuentran en posición de estar inmersos en una espiral de factores que podrían ser desencadenantes de exclusión múltiple.

Las políticas públicas deberían, en nuestra opinión, incorporar la discapacidad como elemento transversal y focalizarse en entornos sociales y educativos desde las épocas más tempranas, pues se ha comprobado en diversas investigaciones la gran influencia de los aprendizajes en la escuela y en el entorno familiar para generar actitudes positivas hacia la discapacidad. Si la educación y los principios morales del agente van ligados a una sensibilización hacia la discapacidad esto fomentará que ese individuo promueva entornos laborales inclusivos en su centro de trabajo porque ya le viene condicionado y predispuesto a ello.

\section{Referencias bibliográficas}

Ajzen, I. (I99I): “The theory of planned behavior”, Organizational Behavior and human Decision Processes, 50: I79-2II.

Banco Mundial y Organización Mundial de la Salud (20I I): Informe mundial sobre discapacidad. Malta: OMS y Banco Mundial (en línea). http://www.who.int/disabilities/ world_report/20I I/es/index.html, acceso el 23 de junio de 2014 .

Comisión Europea (2010): Europa 2020: Una estrategia para un crecimiento inteligente, sostenible e integrador. Bruselas, 3.3.20Io (en línea). http://ec.europa.eu/ commission_20IO-20I4/president/news/ documents/pdf/20100303_I_es.pdf\%.

De Lorenzo, R. (2004): “El futuro de los discapacitados en el mundo: el empleo como factor determinante para la inclusión”, Revista del Ministerio de Trabajo y Asuntos Sociales, 50: $73-89$.

Escarbajal Frutos, A. y Izquierdo, T (20I3): "Psycho-social perceptions about exclusion that determine social and occupational inclusión", Revista de ciencias sociales, I9(I): I3-2I.
España. Real Decreto I276/20II, de I6 de septiembre, de adaptación normativa a la Convención Internacional sobre los derechos de las personas con discapacidad, Boletín Oficial del Estado, núm. 224, de 17 de septiembre de 20I I, pp. $98872-98879$.

España. Real Decreto Legislativo I/20I3, de 29 de noviembre, por el que se aprueba el Texto Refundido de la Ley General de derechos de las personas con discapacidad y de su inclusión social, Boletín Oficial del Estado, núm. 289, de 3 de diciembre de 20I3, pp. 95635-95673.

España. Ley I3/I982, de 7 de abril, de integración social de los minusválidos, Boletín Oficial del Estado, núm. I03, de 30 de abril de I982, pp. I I IO6-I I I I2. [Disposición derogada].

España. Constitución Española, Boletín Oficial del Estado, núm. 3I I , de 29 de diciembre de 1978.

European Foundation for the improvement of living and working conditions (1995): Public Welfare Services and Social Exclusion: The development of consumer-oriented initiatives in the European Union, Dublín: Loughlinstown House. 
Instituto Nacional de Estadística (20I3): Notas de prensa: El empleo de las personas con discapacidad. Explotación de la Encuesta de Población Activa y de la Base Estatal de Personas con Discapacidad. Año 2012, Madrid: INE (en línea). ‘http://www.ine.es/prensa/ np82I.pdf».

Ministerio de Trabajo e Inmigración y Ministerio de Sanidad y Política Social (2009): Estrategia global de acción para el empleo de personas con discapacidad 2008-20I2, Madrid: MTIN y MSPS (en línea). http://www.empleo.gob. es/es/sec_trabajo/estrategia_global_personas_ discapacidad_2008_20I2.pdf, acceso el Io de mayo de 2014 .

Muñoz-Cantero, J. M.; Novo-Corti, I. y RebolloQuintela, N. (20I3): “Análisis de las actitudes, de los jóvenes trabajadores del sector textil hacia la discapacidad: diferencias por razón de género", Revista de Investigación Educativa (RIE), 3I(I): 93-I I 5 (en línea). http://dx.doi. org/IO.60I8/rie.3I.I.I 5 I 8 I I', acceso el I2 de abril de 2014 .

Naciones Unidas (2006): Convención sobre los derechos de las personas con discapacidad, Nueva York: Naciones Unidas (en línea). http://www.un.org/disabilities/documents/ convention/convoptprot-s.pdf).

Novo-Corti, I. \& López, D. (20I3): “La búsqueda de trabajo normalizado para las personas con discapacidad: un análisis del papel de los compañeros como factor de inclusión laboral", Congreso REPS 2013, Universidad Alcalá, Madrid: panel 4 (en línea). http://www3.uah. es/congresoreps20I3/Paneles/panel/4sesion2/ isabel.novo.corti@udc.es/TCNovoCorti.pdf, acceso el I de junio de 2014.

Novo-Corti, I.; Muñoz-Cantero, J.M. y CalvoPorral, C. (20I I): “Analysis of attitudes towards disability among university students: a focus on the theory of reasoned action", Relieve, I7(2): I-26 (en línea). http://www. uv.es/RELIEVE/vi 7n2/RELIEVEvi 7n2_5.htm', acceso el 2 de febrero de 2013.

Obra Social "la Caixa" (20I2): Declaraciones Carlos Pereda coautor Estudio Social n.33: Discapacidades. Fundación "la Caixa" (en línea). http://prensa.lacaixa.es/obrasocial/ estudio-social-33-discapacidades-e-inclusionsocial-fundacion-la-caixa-esp_8 8 6-c-I 5456_. html, acceso el I 4 de febrero de 2014.

Pereda, C.; De Prada, M.A. y Actis, W. (20I2): Discapacidades e inclusión social, Barcelona: Obra Social "la Caixa”, Colección Estudios Sociales, 33 (en línea). http://obrasocial. lacaixa.es/deployedfiles/obrasocial/Estaticos/ pdf/Estudios_sociales/vol33_es.pdf, , acceso el I4 de febrero de 2014 . 